\title{
El comportamiento del presupuesto de seguridad durante el periodo 2004-2013, un insumo para el posconflicto
}

\section{Security budget behavior during the period 2004-2013, an input for the post-conflict}

Ginna Alejandra Ordóñez Fuentes

Juan Manuel Ochoa Amaya ${ }^{2}$

\section{Artículo de investigación}

Recepción: 10/12/2020

Aceptación: 06/07/2021

El Conuco es una revista de acceso abierto revisada por pares. (C) $2018 \mathrm{El}$ autor (es). Este es un artículo de acceso abierto distribuido bajo los términos de la Licencia Internacional Creative Commons Attribution 4.0 (CC-BY 4.0), que permite el uso, distribución y reproducción sin restricciones en cualquier medio, siempre que se acredite el autor y la fuente originales.

Consulte http://creativecommons.org/licenses/by/4.0/. ๑OPEN ACCESS

\section{(c) $(1) \ominus$}

\footnotetext{
Economista, email: ginnaalegrandraordonezfuentes@gmail. com; Orcid: https://orcid.org/0000-0003-2320-5466

2 Doctor (C) Estudios Territoriales (Universidad de Caldas); MSc Desarrollo Sostenible y Medio Ambiente (Universidad de Manizales), Economista (UPTC), Docente de Planta Universidad de los Llanos; líder Grupo de Investigación Territorio y Ambiente, email: juan.ochoa@unillanos.edu.co; Orcid:_https://orcid.org/0000-0001-7198-0577
}

Como citar este artículo / To reference this articcle: comportamiento del presupuesto de seguridad durante

C: (investigación, economía y sociedad), 3(1), pp. 1-14.
DOI: https://doi.org/10.22579/2619-614X.767

\section{Resumen}

En el periodo 2004-2013 se presentaron cambios en el presupuesto destinado a seguridad y defensa, con rubros especiales para la consecución de resultados de primera necesidad en ese momento, como el de la protección del territorio nacional y sobre el cual se desarrollaron dos procesos de paz y una política intensiva de seguridad.

El trabajo acá presentado deja entrever que dentro de un mundo globalizado es muy difícil alcanzar Seguridad y Defensa al mismo tiempo para una nación, si no se cambia el comportamiento del ser humano, es decir, la formación en valores con principios de autorregulación, para de esta forma favorecer la gobernabilidad y la legitimidad.

Palabras Clave: Comportamiento, seguridad, gobernabilidad, legitimidad, eficacia, eficiencia.

Clasificación JEL: A1; H1; H7

\section{Abstract}

In the period 2004-2013 changes were made in the budget for security and defense, with special areas for achieving results staple at that time, such as the protection of national territory and which were developed two processes intensive peace and security policy. 
The work here presented suggests that in a globalized world is very difficult to achieve security and defense at the same time for a nation if human behavior, ie, the formation of values with principles of self-regulation is not changed, and in this shape promote good governance and legitimacy.

Keywords. Performance, security, governance, legitimacy, effectiveness, efficiency.

\section{Introducción}

La historia reciente del conflicto en el ámbito social dio pasó a las Fuerzas Armadas Revolucionarias de Colombia Ejército del Pueblo (FARCEP), que como profesa el Gral. Jorge Enrique Mora Rangel para describirlas, fueron una "guerrilla que siempre estuvo interesada en mostrar la ausencia, ineficiencia y abandono en que el Estado los mantenía" (Escuela Superior de Guerra de Colombia, 2008, p.5).

Así, esta estructuración del conflicto en Colombia se ha tratado de contener mediante el fortalecimiento de las fuerzas militares, fuerzas que en los últimos años mediante inyección al presupuesto destinado a la defensa y seguridad, redireccionó la política de defensa, enfocándose en la recuperación del territorio.

En términos técnicos y financieros para adentrarnos en el aterrizaje dentro de este campo de la continuidad del conflicto y el intento gubernamental por contenerlo, La división fiscal de las Naciones Unidas quien ha sugerido, "Un presupuesto por programas y actividades es un sistema en que se presta particular atención a las cosas que un gobierno realiza más bien que a las cosa que adquiere...lo que no queda claro en los sistemas presupuestarios tradicionales es esta relación entre cosas que el gobierno adquiere y las cosas que realiza”. (Martnerm, 2004, p. 89)
Lo cual definitivamente crea un interrogante frente a la efectividad sobre la aplicación de políticas en términos de defensa y seguridad, trayendo a colación el comportamiento del índice de criminalidad entre los años 2002 y 2013 que a pesar de estar respaldado por un presupuesto estable y constante, no se ve reflejado en el comportamiento estable y constante en su índice de criminalidad.

\section{Marco teórico}

Max Weber y su Concepción Sobre el Control Social. La política en Seguridad y Defensa implementada en los últimos tres gobiernos de Colombia a partir del 2002 hasta el 2014, se intentó mostrar como el poder de organización de las fuerzas militares sobre el orden social al inicio de su implementación. Lo cual es puesto en consideración debido a los resultados negativos de los últimos años colocados a la luz de los principios de Eficacia, Eficiencia y Efectividad, los cuales parecen no dar resultados pese a la inflexión presupuestal de la que goza el presupuesto de Defensa y Seguridad. Por lo cual realizar análisis comparativos entre recursos invertidos, resultados, e ideologías sobre las actuaciones del Ministerio de Defensa y Seguridad se convierte en punto esencial, para determinar su interrelación.

Las formas de vigilancia, control, disciplinamiento...muchas veces son justificadas y racionalizadas en aras de la seguridad, la sanidad, el funcionamiento eficaz y moral...actualmente ningún estado nacional prescinde de un circuito institucional de procuración de justicia y seguridad pública. El Estado moderno sigue siendo el que garantiza la seguridad y monopoliza la violencia legítima, por lo que requiere de instituciones especializadas para ello. Instituciones de seguridad que también inciden sobre el entramado social, cultural e institucional y viceversa. (Payá, 2001, p. 92) 
Lo anterior dista de lo que Pecaut refiriéndose a la Justicia Transicional Colombiana llama "suerte de vulgata como recurso discursivo con eficacia simbólica pero sin capacidad transformadora, con capacidad de volverse creíble porque responde a los deseos de muchos colombianos" debido al hecho de estar lejos de mirar el pasado para proyectase en el futuro, por encontrarse en un régimen violento, en el que diversos grupos ilegales se disputan territorios y zonas estratégicas, dejando desarraigo, destrucción, muerte y miedo a su paso" (Muñoz \& Bondia, 2009, p. 47).

El siguiente hecho recurrente hace necesario acudir a Weber para determinar el tipo de liderazgo al cual se adapta el Ministerio de Defensa y Seguridad y por consiguiente todas sus dependencias. Este distingue tres tipos de dominación legitima: 1. De carácter racional. 2. De carácter tradicional y 3. De carácter carismático la cual descansa en la entrega extracotidiana a la santidad, heró́smo o ejemplaridad de una persona y a las ordenaciones por ella creadas o reveladas" (Weber, 1984, p. 172).

Así, esta clasificación enmarcaría a las dos instituciones que monopolizan las armas oficialmente en Colombia en una Legitimidad de Carácter Carismático; esto y la visión de Max Weber quien determinaba el uso de las capacidades tecnológicas como un contrastante de dominio de la naturaleza y sumisión a los procesos de eficientización, a costa de los valores profundos que arraigan al hombre con su entorno (Payá, 2001, p. 85), infiere que la justificacion del fortalecimiento de las fuerzas armadas apartir de dotacion de equipo y armamento militar, aumenta mas su ineficiencia respecto al indice de criminalidad. Incluso en contravia via de los principios de metodologias del FMI, respecto a evaluar el gasto público por finalidad y función, asi como la necesidad de hacerlo en términos de flujos monetarios anuales y no de activos físicos y financieros acumulados en distintos períodos más prolongados (Naciones Unidas, 2011, p. 15).

Reflejando esta situación un factor de ineficiencia, determinado en la mera voluntad de producir resultados, estableciendo que la obligación del estado de brinda condiciones de seguridad no es inherente a la institución.

Saliendo esta institución de lo que el capitalismo identifica con la aspiración a la ganancia lograda con el trabajo capitalista incesante y racional, la ganancia siempre renovada, a la rentabilidad. Dentro de una ordenación capitalista de la economía, todo esfuerzo individual no enderezado a la probabilidad de conseguir rentabilidad está condenado al fracaso (Weber, 2009, p. 17). Con lo cual se cobijaría el fracaso de las fuerzas de la defensa y seguridad como monopolizador de la violencia, debido a la ausencia de ganancia renovada o rentabilidad con respecto a la consecucion de sus fines, aclarando que un mundo globalizado la ganancia no es solo consecuencia de produccion material, si no ademas produccion intelectual o en terminos contables el Know-how.

Aunque las instituciones públicas están sujetas al juego de la oferta y la demanda, y cabe juzgarlas bajo los principios del mismo mercado, "La actividad económica consiste en guiarse en todo momento por el cálculo del valor dinerario aportado y el valor dinerario obtenido al final, por primitivo que sea el modo de realizarlo" (Weber, 2009, p.20).

Por lo tanto es de interrogarse sobre la eficiencia en el uso de los recursos en los procesos internos de las fuerzas militares. $\mathrm{Y}$ en el entendido de que las instituciones están compuestas por individuos, es pertinente acudir a las concepciones naturales de la eficiencia de los individuos, para indagar sobre las afectaciones en que pueden in- 
fluir las acciones individuales dentro de la consecución de objetivos generales de la institución. Weber (2009) enunciaba "la actividad industrial permite a sus miembros más dotados satisfacer una ambición que no pueden colmar sirviendo al Estado" (p.41).

Entre dejando ver una falta de desenvolvimiento capitalista o económico en el pensamiento militar, policivo y burocrático, que debería exigirse. Abriendo espacio al fracaso de las instituciones siempre que se trata de conseguir productos que exigen un trabajo cualificado, o el empleo de máquinas costosas y fácilmente inutilizables, o, en general, un mayor esfuerzo de la atención y una mayor iniciativa (Weber, 2009, p.72). Evitando dejar a la opinión pública como único legitimador de los buenos o malos resultados obtenidos.

Weber (2009) declamaba "en las acciones civiles es bueno ser como la mayoría; en las religiosas, como los mejores" (p.225), por lo cual es difícil pretender que las instituciones compuestas por personas que carecen de estos principios puedan a través de sus principales responsables incidir en las buenas acciones de sus subordinados. Ejemplificación de esto en "falsos positivos, dolorosos homicidios extrajudiciales que muchos han advertido era una política de Estado" (Semana, 2014).

Tanto que sería pertinente retomar en lo que los sociólogos insistieron "las prácticas de control social no deberían partir directamente del estado (quien puede aplicar la violencia), sino precisamente de la dinámica social, o sea, a partir de la integración de la sociedad, a partir de los procesos de socialización, que son los que instituyen a los sujetos dentro del orden de lo permitido. En síntesis, una cohesión de la sociedad que otorgue una estabilidad, gracias a los límites y controles autoimpuestos por los propios individuos que integran aquella (Payá, 2001, p. 96).
Así, retomar los lineamientos modernos del Estado basados en "políticas públicas que siguen procesos de optimización y racionalización de las relaciones sociales, apoyadas en el discurso de la ilustración como el único capaz de sostener aquellos hombres y mujeres capaces de desterrar para siempre la precedencia de demonios" (Payá, 2001, p. 85) desde la perpectiva Weberiana. Argumentos coherente con el cambio de politica nacional en base a la seguridad por la apertura a la ideologia del dialogo y de la paz.

Donadío y la Perspectiva sobre la Estructura Social que incide en el Comportamiento del Presupuesto de la Defensa y Seguridad de los Países. Los antecedentes teóricos que analizan el presupuesto de los estados en defensa y seguridad a nivel de América Latina se centran en las definiciones de "estado colonial el que organizó la región; definiendo que fuero las precarias administraciones de las nuevas repúblicas las que, poco a poco, tomaron el control territorial y organizaron a la sociedad, la cual en ese tiempo se dividía en dos grupos: los dominantes, la pequeña elite de letrados y caudillos armados, y los dominados, sus seguidores o, simplemente, el resto de los habitantes" (Donadio, 2004, p. 13).

Con esto se empiezan a vislumbrar las razones para generar afectaciones dentro del presupuesto de defensa de un estado. Actualmente el medio investigador reconoce a El Estado "gendarme" con las funciones financiera (recaudación de impuestos), justicia, seguridad y defensa. Pero a la vez reconoce El Estado ha dejado de ser el monopolizador de la violencia (Donadio, 2004, p.18).

Debido a que hay zonas donde la presencia estatal solo se reduce a un contingente de la fuerza militar. Y por otro lado la alianza táctica o específica entre narcotraficantes y sectores subversivos o insatisfechos en más de un 
caso obliga a la acción conjunta de policías y militares. A ello se agrega el temor de la acción de grupos que practican el terrorismo como forma de lucha en el marco de un mundo globalizado (Donadio, 2004, p.18).

Lo cual se intenta contrarrestar mediante financiación. Donadío (2004) define esta financiación con la necesidad de "dotar de misiones y objetivos el instrumento militar" (p.84), pero los fines financiados en Colombia obedecen más a problemas históricos acumulados dentro de la administración, como el pasivo pensional.

La investigación reciente cuenta con el Análisis del gasto militar desde la perspectiva de la Economía de la Defensa, el caso colombiano 19502006. En el cual comprueban cómo el gasto se mantuvo por debajo del promedio regional a través del siglo XX, a la vez que el gasto de defensa y seguridad de este país no se correlaciona con el conflicto interno y la trayectoria que sigue es aleatoria. Estableciendo una generalidad trascendente "la Política de seguridad y defensa es producto de eventos coyunturales y no el fruto de una planificación del primer bien público que debe entregar un Estado a sus ciudadanos" (Grautoff \& Chavarro, 2009, p.202).

Estas generalidades también han sido determinadas en el programa de Apoyo a la Descentralización y el Desarrollo Local para la Paz, ejecutado por la cooperación alemana para el Desarrollo, quienes buscaron generar espacios de reflexión sobre las políticas públicas nacionales con el ánimo de aportar insumos que consoliden las bases del desarrollo y la paz (GTZ; FESCOL, 2003 pp.44-46). Identificando por tanto la forma en la cual se evoluciona hacia organizaciones complejas con miras a reducir esas incertidumbres, como la organización de grupos armados y la moderación de los comportamientos oportunis- tas de miembros de la sociedad ante los vacíos de gobernabilidad y ausencia estatal (Forero, Cardona, \& Cordona, 1999).

Las distintas representaciones de las instituciones oficiales se han inmerso en lo que Collier (como se citó Romero, 2011, p.82) llama "vacío de gobernanza política y económica" identificando el credo (Objetivos políticos que motivaron el conflicto violento) y la codicia (medios susceptibles de ser capturados y controlados para obtener beneficios de la continuación del conflicto) que es finalmente lo que conlleva a un aumento en el gasto en seguridad y defensa.

Este mismo lineamiento lleva a Gutiérrez y Jaramillo (2003) a acuñar la noción de "pactos paradójicos" entre actores y agentes ubicados en ambos lados de la línea que separa lo legal y lo ilegal. Para asegurar procesos continuados de captura de rentas en ambientes donde no hay condiciones para que opere una lógica de "Economía de guerra" (Romero, 2011, p. 84).

Adicional Pearce (2005) se acoge también a esta corriente y denomina como el modelo de "gobernabilidad selectiva", a través del cual las elites políticas de orden nacional ceden el control de extensas porciones del territorio y la población a estructuras locales de poder. A fin de administrar represión a través de actores al margen de la ley, con capacidad demostrada para controlar los factores de "desorden" (Romero, 2011, p. 86).

En todo caso,

Todos estos bajo la visión psicológica de la ciencia del comportamiento, porque determinar un fenómeno económico de forma acertada debe observar el comportamiento del hombre en el tiempo no solo en el momento precedente, sino también durante toda su vida, puesto que el hombre es un sistema crono-holístico (Devereux, 2003, p. 28-29). 


\section{Antecedentes}

Desarrollo Histórico del Presupuesto de Defensa y Seguridad en Colombia. "El Presupuesto ha desempeñado un papel puramente pasivo y las presiones sociales y políticas que se descargan sobre él han definido a la postre su estructura" (Martnerm, 2004, p. 7). Lo cual se ve reflejado en el desarrollo histórico del conflicto armado en Colombia, de la siguiente manera, en 1933 durante el conflicto con Perú, el porcentaje del gasto militar con respecto al PIB fue del 3\%; con el mandato de Laureano Gómez el conflicto bipartidista se agudizó tras la muerte de Jorge Eliecer Gaitán y el gasto en defensa con respecto al PIB alcanzó un $2 \%$, después de los cincuenta uno de los mayores aumentos del gasto en defensa está relacionado con la nivelación salarial de la Policía y las Fuerzas Militares (en menor medida) en la década de los noventas llegando a tener un 3.1\% con respecto al PIB (Valecia, 2012).

Así, para el periodo (1998-2002), durante el gobierno del presidente Pastrana, en medio de la implementación del Plan Colombia, el gasto en defensa creció más que en décadas anteriores, situación contraria a lo presentado en insurgencia, pues el auge de las Farc hizo mella en 20 municipios del país, llegando a tener cerca de 20.000 hombres en su pie de fuerza (Valencia, 2012).

De la anterior descripción histórica se podría deducir que aunque ha habido un aumento en el presupuesto de la defensa y seguridad, no se habían obtenido resultados realmente relevantes respecto a la disminución del conflicto.

Ahora, debido a la pérdida de control sobre la población y el territorio, para el 2002 la lucha antisubversiva se convirtió en una política de estado, y con en el Plan de Consolidación del segundo periodo presidencial del Álvaro Uribe, se buscó la derrota militar y obligar al grupo armado a negociar su futuro político con el gobierno. Todo este cambio de política naturalmente acompañado de un aumento en el presupuesto de defensa y seguridad (Escuela Superior de Guerra de Colombia, 2008).

Este comportamiento de las acciones del estado conlleva a repasar los conceptos de presupuesto y sus efectos sobre la política pública. La Comisión de Organización Administrativa del Estado de Maryland, define "el presupuesto tradicional como la ilusión de precisión y control legislativo, pero es en los hechos confusos, que se frustran los intentos de interpretar lo que el presupuesto realmente significa en términos de la actividad del estado" (Martnerm, 2004, p. 291). Lo que genera un interrogante a partir del hecho de haber perdido esa ilusión de precisión y control legislativo de un a año a otro, con el aumento disparado de los índices de criminalidad para 2012.

La estructura burocrática de la defensa y seguridad abre espacio a Martnerm (2004) quien sostiene "el problema está en que muchas de las cosas que el gobierno hace no son significativas como una medida para producir resultados dentro de un programa" (p. 293); estructura burocratica que ademas no causa la ilusion de precision y control legislativo.

Entonces el interrogante se materializa cuando al observar el comportamiento del presupuesto para la seguridad y defensa nacional durante tres gobiernos con un presupuesto similar, los resultados de la gestión se contrastan particularmente del 2010 al 2012.

Así, el presupuesto destinado a la defensa tiene un comportamiento con un aumento relativo promedio entre 2001 y 2013 del 6,1\%, dentro de este cabe destacar que en el segundo periodo del 
gobierno del Uribe Vélez 2006-2010 tuvo un aumento máximo para el año 2008 respecto al año anterior de alrededor del 16,7\%, visiblemente por encima del promedio para el periodo comprendido entre 2001-2013 teniendo para el 2008 un monto total de veintiún billones de pesos.

Con respecto a los resultados y efectividad de las políticas aplicadas con estos recursos, el primer gobierno del Uribe Vélez inició en el 2002, recibiendo un índice de criminalidad del 1,5, éste disminuyó significativamente para el 2006 situándose en el -0,42, momento en el cual fue reelegido el gobierno anterior; al finalizar el segundo periodo del Álvaro Uribe Vélez entrega un índice de criminalidad situado para 2010 en el $-0,84$.

Estos resultados fueron posibles según Pérez y Valbuena (2014) debido a los cambios legislativos realizados como parte de la fuerte política de seguridad, debilitando drásticamente a las organizaciones criminales. Comprometiéndose el gobierno a combatir militar y financieramente sus estructuras. Todo demando la necesidad de declarar el estado de conmoción interior con el fin de adoptar las medidas necesarias para reducir los altos niveles de violencia.

A raíz del propósito superior adaptado desde el 2006 "Contribuir a la gobernabilidad democrática, la prosperidad colectiva y la erradicación de la violencia, mediante el ejercicio de la seguridad y la defensa, la aplicación adecuada y focalizada de la fuerza y el desarrollo de capacidades mínimas disuasivas," (Ministerio de Defensa, 2011, p.31). Dentro de la Política Integral de Seguridad y Defensa para la Prosperidad - (PISDP).

Estos resultados contrastan con un índice de criminalidad para el 2012 de 1,5 para el primer gobierno de Juan Manuel Santos, reflejando este último un retroceso de 9 años de una política que mantenía los índices de criminalidad controlados, y señala la falta de coherencia de los resultados frente al gasto constante y estable del presupuesto del Ministerio de Defensa Nacional, el cual termino en 2013 con 27 billones de pesos (Ministerio de Hacienda y Credito Pùblico, 2014). $\mathrm{Y}$ en contradicción del principio de irreversibilidad, esperando a su vez alcanzar la ilusión que en Colombia se de una prosperidad para todos con más empleo, menor pobreza y una mejor seguridad (Ministerio de Hacienda y Credito Publico, 2011). El cual fue la justificación esencial para la adición presupuestal para Defensa y Seguridad periodo 2011-2014 en el documento CONFIS del 2011.

\section{Metodología}

Se desarrolló la metodología de marco lógico, la cual es pertinente, ya que "ordena, conduce y orienta las acciones hacia el desarrollo integral de un país, región, municipio o institución" (Ortegon, \& Prieto, 2005, p.9). Con base en este trabajo se describirán la situación actual, para establecer la pertinencia del proyecto en medidas de eficiencia, eficacia y efectividad, para finalmente describir el comportamiento del presupuestó periodo 2004-2013. Para la producción de indicadores se acogió la Guía para la construcción de Indicadores de Gestión, elaborada por el Departamento Administrativo de la Función Pública.

El método empleado fue deductivo-inductivo con la finalidad de entrar en un análisis soportado por la identificación de cada una de las partes que caracterizan la realidad; de este modo se establecieron las relaciones causa-efecto entre los elementos que componen el objeto de investigación (Méndez, 1995), para al final terminar con una síntesis a partir de la interrelación de los elementos que identifican su objeto (Méndez, 1995). 
Los indicadores fueron enfocados a seguir el método deductivo estableciendo situaciones generales que explican situaciones particulares contenidas explícitamente en la situación general. Indicando la afectación en las actividades principales que permiten cumplir con los objetivos de la Política Integral de Seguridad y Defensa para la Prosperidad - (PISDP).

Se realizó la selección de aquellas actividades que se consideran prioritarias. Las cuales contribuyen directamente a la gobernabilidad, la prosperidad, erradicación de la violencia, y el desarrollo de capacidades discusivas. Definiendo la Incidencia de Fortalecimiento de Seguridad Pública, la cual mide las actividades que contribuyen a la gobernabilidad, prosperidad, y erradicación de la violencia, y la incidencia de los Resultados Operacionales de la Fuerza Pública, lo cuales miden el Desarrollo de capacidades disuasivas.

\section{Resultados}

\section{Análisis de Indicadores de Eficacia.}

1. Focalización: Indicador por hectárea (ha) del territorio continental colombiano afectado en promedio por Actos de Terrorismo. De un total de 114.174.800 ha de territorio continental colombiano, con una política intensiva de seguridad y defensa aplicada a un periodo analizado de 10 años entre el 2003 y el 2013, tuvo como resultado de mayor focalización el año 2007 con la realización de 387 Actos terroristas, afectando en promedio 38.700 ha. Y menos focalización el año 2012 con la realización de 894 Actos terroristas, afectando en promedio 89.400 hectáreas afectadas.

Esto lleva a que las fuerzas armadas aumenten el número de hombres pero con un material bélico no adecuado para la defensa y disuasión de amenazas externas; la elevación del gasto de defensa y seguridad aumenta la ineficiencia del sector pu- blico mientras los indicadores sociales se deterioran, los gobiernos pierden legitimidad y emplean la fuerza militar para cumplir su misión de defensa externa, sin duda ineficiente; y se la conduce al control de cataclismos sociales aumentando la violación de derechos civiles, lo que a la postre generan un aumento del gasto de defensa y seguridad. (Grautoff \& Chavarro, 2009)

2. Cobertura: Indicador Porcentaje \% del territorio colombiano afectadas por actos de terrorismo. De un total de 114.174.800 hectáreas de territorio continental colombiano, con una política intensiva de seguridad y defensa aplicada a un periodo analizado de 10 años entre el 2003 y el 2013, tuvo como resultado de mayor cobertura el año 2007, viendo afectado por actos de terrorismo el 0,00034\% del territorio continental. Y menos cobertura el año 2012, viendo afectado por actos de terrorismo el $0,00078 \%$ del territorio continental.

El porcentaje de territorio que goza con cobertura es irrelevante y prácticamente imposible de alcázar a niveles presentables. Una alternativa más eficaz frente a la seguridad, debería focalizarse al uso de proyectos de inversión en construcción de obras públicas, lo cual reduce sensiblemente los costos de transporte y comunicaciones, impactando positivamente el sector primario de la economía del cual dependen las economías con mercados emergentes. (Grautoff \& Chavarro, 2009).

3. Resultado Final: Indicador Porcentaje de ciudadanos colombianos afectados por los actos de terrorismo. Finalmente, como resultado final para determinar la eficacia de la política de seguridad y defensa aplicada a un periodo analizado de 10 años entre el 2003 y el 2013, represento mayor eficacia para el año 2007, con una población de 43.926.930 hab, afectando al 0,0009\%, con la realización de 387 actos terroristas. Pre- 
sentándose con menor eficacia en el año 2012, con una población de 46.581 .820 hab, afectando al $0,0019 \%$, con la realización de 894 actos terroristas. A la "fecha se mantiene y que responde al aumento secular del pie de fuerza, que llega a representar un 5,2\% con respecto al PIB" (Grautoff \& Chavarro, 2009, p.221). Lo anterior indica que los actos de terrorismo no alcanzan a un porcentaje representativo de la población, siendo la principal justificación para una alta inversión en políticas contra subversivas.

Lo que querría decir que el aumento en el gasto de seguridad y defensa no repercutiría en la eficacia del servicio, si no cubriendo problemas históricos de la institución por lo que no se observan resultados considerables respeto a la cobertura en territorio y población. Abriendo mucho espacio a los vacíos de gobernanza.

\section{Análisis de Indicadores de Eficiencia.}

\section{Costo la Defensa y Seguridad en Prome-} dio para cada Ciudadano: Con un presupuesto destinado a la seguridad y defensa para 2013 de $\$ 27.1$ billones, con una población de 47.121.090 hab, cada colombiano contribuye en promedio con $\$ 575.114$ anualmente. Un valor de $\$ 47.926$ mensual, lo que es equivalente al $7.7 \% \%^{3}$ del smmlv mensual, lo cual comparado con el aporte mensual del $4 \%$ a salud que deben hacer todos los empleados formales, deja en consideración la baja financiación de la que sufre el sector salud al enviar más recurso a un servicio costoso e ineficaz como la seguridad y defensa. "La búsqueda de beneficios conduce a una política nacional depredadora" (Veblen, 1965, 328), refiriéndose a lo que las grandes fortunas exigen. Prueba de

3 Todos los datos monetarios se encuentran en precios constantes del 2014, por lo cual se toma para el cálculo del porcentaje mensual la base es $\$ 616.000$ smmlv 2014, y el aporte de $4 \%$ a salud para empleados para 2013 según Circular Externa 00101 de 2007. ello el alto costo de ofrecer seguridad y defensa a una pequeña proporción de propietarios de propiedad privada. Sin ofrecer respuesta a las demandas en servicio sociales, esenciales para la vida humana y la integridad del ser humano.

\section{Costo total de la Defensa y Seguridad en} Promedio para cada hectárea de territorio Continental: Con un presupuesto destinado a la seguridad y defensa para 2013 de $\$ 27.1$ billones, con una superficie territorial continental de 114.174.800 hectáreas, cada hectárea le cuesta al Estado en términos de seguridad y defensa en promedio $\$ 237.355$, la inequidad en el uso de los recursos públicos se evidencia en el hecho de que el 52,2\% del área de territorio continental de Colombia pertenece al 1.15\% ubicados como los grandes propietarios poseedores de la tierra (PNUD, 2011, p. 48), es decir la mitad de este gasto excesivo solo está dirigida a beneficiar la propiedad privada de 541.893 habitantes de un total de 47.121.114 población colombiana.

Por lo cual el gasto en defensa y seguridad no debería estar justificado en la consolidación de todo el territorio pues el $52 \%$ de este es propiedad privada de grandes poseedores de la tierra. Por lo cual debería deducirse al límite que permita el desarrollo de la institucionalidad.

Y, aun así, la efectividad de la política ni siquiera es bien reconocida por los escasos privilegiados, como se deja ver en Otero (2008) "estaba convencido de la ineficacia de las fuerzas militares y su principal objetivo era la defensa de la vida, honra y bienes de los propietarios de la región", una de las justificaciones para el nacimiento de los cuerpos paramilitares (p.56).

\section{Costo de cada décima del índice de Cri-} minalidad: De un presupuesto destinado a la defensa y seguridad para 2013 de $\$ 27.1$ billones, con un índice de criminalidad 1,37, cada décima 
del Índice tuvo un costo de $\$ 1,9$ billones. En promedio para el periodo analizado 2003-2013 cada décima del índice de criminalidad le costó al estado $\$ 4,9$ billones de pesos en promedio.

Por lo cual el hecho de tener retrocesos en los resultados, con el agravante de que no solo se mantiene el monto del presupuesto a la seguridad, sino que además se hicieron inversiones en armamento militar y dotación; evidencia una clara ineficiencia en racionalidad económica y más aún en la obligación moral de entregar compromiso individual al accionar de una institución. "El gasto fiscal es inflexible, en relación con el gasto social existen rublos como el situado fiscal que hace inviable que este se vea modificado por políticas de defensa y seguridad" Grautoff \& Chavarro, 2009, p. 207).

Lo que obligaría al desarrollo intelectual dentro de la institución para producir valor agregado, que mejores la eficiencia de los procesos internos. Para disminuir los costos por la pérdida de resultados para la nación.

Donadío (2004) define esta financiación con la necesidad de "dotar de misiones y objetivos el instrumento militar, estableciendo las hipótesis de su empleo" (p.84), pero los fines financiados en Colombia obedecen más a problemas históricos acumulados dentro de la administración.

\section{Análisis de Indicadores de Efectividad.}

\section{Afectación anual Índice de Criminalidad:} El 2004 respondió a la política de choque implementada en los primeros años de la PISD con la disminución de 1,27 unidades respecto al año anterior dentro del índice general. Mientras que para el año 2012 hubo una situación de retroceso con la que se aumentó 1,34 unidades respecto del año anterior dentro del índice de criminalidad. Con lo cual se demuestra la falta de planificación y efectividad de la política de seguridad. Debido a la imposibilidad de conseguir resultados de largo plazo con los que la población civil legitímese el suministro del servicio de seguridad en Colombia.

2. Porcentaje de variación en el Índice de Criminalidad: Reafirmando la situación anterior se encuentra el porcentaje de variación que para 2004 registro una disminución porcentual de $126,97 \%$ con lo cual cayó abruptamente el índice de criminalidad.

Pero aumentado en la misma intensidad el incremento porcentual del índice de criminalidad, abarcado134, 42\%, más del 100\% para el 2012, con lo que se deja serios cuestionamientos en la capacidad del servicio de defensa y seguridad para dar abasto a la demanda. Además de generar interrogantes como el procedimiento interno para evaluar la efectividad de su rendimiento. Debido a que no se tomaron las medidas pertinentes desde dentro para disminuir el impacto negativo sobre este índice. Ante la falta de efectividad, es de aclararse que la reducción del gasto no necesariamente acelera el crecimiento económico. $\mathrm{El}$ aumento del gasto en conclusión establece la correlación que sigue este con respecto al crecimiento económico y no su efectividad (Grautoff \& Chavarro, 2009). Pero si deja expuesta, la soberanía, la independencia, la integridad del territorio nacional y del orden constitucional.

\section{Recepción del Servicio de Seguridad y} Defensa por cada Ciudadano: Así también, aunque para el año 2005 los ciudadanos recibieron una mayor cantidad del servicio de seguridad y defensa, disminuyendo en promedio hasta $-0,00041 \%$ su recepción de inseguridad.

Para el 2012 el porcentaje aumento, haciendo que avanzara positivamente hacia una recepción 
de inseguridad, recibiendo menos seguridad de la que venían recibiendo con una sensación de inseguridad de 0,00020\%.

En cualquiera de los dos casos se evidencia la incapacidad de brindar efectividad para un ciudadano promedio, ya que los ciudadanos corrientes tienen una concepción material casi nula de seguridad y defensa y el porcentaje de población que la recibe por ejemplo para el 2005 año en el cual se sostuvo un índice presentable, apenas tenía capacidad de ser efectivo satisfaciendo a 17.469 ciudadanos. Los riegos para el simple ciudadano empiezan a estar presentes en la cotidianeidad y es necesario vivir con este factor y acomodarse de la mejor forma posible a él (Otero, 2008).

4. Variación en la Recepción de Seguridad: Los ciudadanos en el 2009, recibieron 175,73 unidades de seguridad debido a la acumulación de año a año.

En contraste se encuentran también similitudes entre la seguridad recibida y la recepción de seguridad. Coincide también en el 2012 donde las personas que recibieron menos seguridad están predispuestas a sentir mucha inseguridad debido a la variación decimal tan amplia de un año a otro, ubicándose en -236,59 lo que representa recepción negativa frente a la seguridad y defensa.

Así, "el gasto de defensa y seguridad en Colombia se mantiene de acuerdo con la inflexibilidad presupuestal del modelo bipolar" (Grautoff \& Chavarro, 2009, p. 206). Como también el gasto de defensa y seguridad como instrumento central de política que hale al resto del sector productivo de una nación no garantiza el bienestar de la nación (Grautoff \& Chavarro, 2009).

Martnerm (2004) sostiene "El problema está en que muchas de las cosas que el gobierno hace no son significativas como una medida para producir resultados dentro de un programa” (p. 293).

\section{Análisis de Indicadores de Economía.}

\section{Monto total de variación de costos por pro-} cesos internos: Se podría considerar alármate que mientras en el 2008 hubo una fuerte variación de un año a otro de $\$ 3,14$ billones de pesos, ni el 2008 ni el 2009 dieron resultados representativos dentro de los indicadores de eficacia, eficiencia, y efectividad, que evidenciara el aumento significativo en los recursos monetarios inyectados a MDS. La activación del gasto mediante seguridad y defensa solo debería ser usada para "la transmisión contra cíclica de las presiones deflacionarias de las economías" (Grautoff \& Chavarro, 2009, p. 205). Lo cual se aleja de un país como Colombia que actualmente se encuentra en auge económico, siendo en palabras del Dr. Juan Manuel Santos "la tercera economía del mundo".

\section{Porcentaje de Defensa y Seguridad sobre} el Gasto General de la Nación: Es el único resultado a lo largo del análisis que guarda coherencia, observando una tendencia creciente en el presupuesto destinado a seguridad y defensa, pero contrastando con una tendencia negativa en relación de este sobre el gasto general de la nación. Debido a hechos coyunturales que han desligado algunas actividades de la seguridad y defensa y otros servicios como la inteligencia y algunos entes investigativos que se financian fuera de este rubro. "La productividad marginal captura las condiciones de paz y seguridad que garantizan que los derechos de propiedad serán respetados, y simultáneamente minimizan los costos de transacción" (Grautoff \& Chavarro, 2009, p. 204). Lo cual en este caso de análisis, si ha capturado las mejores condiciones del entorno, no obedecen a unos resultados de la institución, lo que se evidencia con la falta de eficacia y eficiencia, reflejando así la falta de efectividad en la institución encargada de la seguridad y defensa, 
si no a condiciones generadas por los fenómenos sociales como los acciones paramilitares.

\section{Conclusiones}

Con la investigación se haya la necesidad de medir de forma diferente los resultados de la Política en Defensa y Seguridad, creando unos indicadores razonables al marco teórico de eficiencia. Con los cuales realmente se abrieran interrogantes que no ocultara la falta de coherencia entre la inversión de recursos públicos frente a la falta de efectividad en la aplicación de la política pública.

Los resultados fueron analizados a la luz de hechos históricos, repetitivos en cada una de las investigaciones Colombianas acerca del gasto del gobierno en Defensa y Seguridad, las que además siguen los lineamientos ya enmarcados de forma general en la historia de la economía, la política y la sociología, respecto al desarrollo de los países y las formas de control social y la imposibilidad en un mundo globalizado para las entidades encargadas de brindar Seguridad y Defensa de satisfacer a los ciudadanos demandantes de este bien público. Siempre y cuando no se acuda a la formación del ser humano desde sus más profundos valores, con el cual se creen principios de autorregulación en los grupos sociales.
Se identificaron los factores que inciden en el comportamiento del Presupuesto de Defensa y Seguridad Nacional en el periodo 2004 a 2013, acudiendo a la fundamentación teórica clásica de Max Weber, abordada desde el caso colombiano y algunas premisas ya documentadas por investigadores nacionales, complementando con la argumentación científica acumulada sobre las situaciones o hechos aceptados socialmente. Lo que se interpone entre la consecución de objetivos de largo plazo de la política pública de seguridad y defensa. Irónicamente las mismas que llevan a sostener un gasto en defensa y seguridad en condiciones de ineficiencia.

Finalmente se determinó que existen hechos recurrentes históricamente que generan ineficiencia en los recursos invertidos sin planificación en el sector de la defensa y seguridad nacional. Así se determinó el comportamiento del Presupuesto asignado a la Seguridad y Defensa del 2004 a 2013, interrelacionando sus sostenimiento financiero respecto a los factores que inciden dentro de este, en la toma de decisiones y consecuencias posteriores en los resultados, sobre la soberania, la independencia, la integridad del territorio nacional y del orden constitucional. 


\section{Referencias}

Donadio, M. (2004). Resdal. Acesso em 05 de 06 de 2014, disponível em http: / / www. resdal.org/assets/libro-guia-presu.pdf

Devereux, G. (2003). De la ansiedad al método en las ciencias del comportamiento. Mexico D.F.: Siglo XXI editores s.a. de c.v.

Escuela Superior de Guerra de Colombia. (2008). El Plan Patriota,base del Plan de Consolidación. Control territorial, legitimidad y consolidación de la Política de Seguridad Democrática, LXXVI, 205, pp. 18-25.

Forero, C., Cardona, G., \& Cordoba, C. (1999). Planeacion Participativa. Colombia: Tercer Mundo Editores.

Grautoff, M., \& Chavarro, M. (2009). Análisis del gasto militar desde la perspectiva de la economia de la defensa: El caso colombiano 1950-2006. Ecos de Economia, [S.l.], v. 13, n. 28, p. 199-237, may. 2011. ISSN 1657-4206.

GTZ; FESCOL. (2003). Ordenamiento Territorial: Reivindicación de la descentralización para el Desarrollo. Bogotá, D.C., Colombia: Grafivisión Editores Ltda.

Gutiérrez, F., \& Jaramillo, A. M. (2003). "Pactos Paradoxais" en de Sousa Santos, B (ed.), Reconbecer para Libertar: Os caminbos do cosmopolitismo multicultural. Rio de Janeiro: civilização Brasileira.

Ladrón de Guevara, L. (1997). Metodología de la Investigación Cientifica. Bogotá: USTA.

Martnerm, G. M. (2004). Planificación y presupuesto por programas. Mexico: Siglo XXI editores.s.a. de c.v.
Méndez, C. (1995). Metodologia: Guia para eleaborar diseños de investigacion en ciencias economicas, contables $y$ administrativas. Bogota: McGraw-Hill Interamericana, S.A.

Ministerio de Defensa Nacional. (2014). Logros de la Politica Integral de Seguridad y Defensa para la Prosperidad. Acesso em 05 de Agosto de 2014, disponível em www.atlas.com.co: www.atlas.com.co/ sia/.../Logros_Sector_Defensa_Junio_2014(16).pdf

Ministerio de Defensa. (2011). Política Integral de Seguridad y Defensa para la Prosperidad.

Ministerio de Hacienda y Credito Pùblico. (2014). Presupuesto General de la Naciòn 2014.

Ministerio de Hacienda y Credito Publico. (2011). Financiacion de la Politica Integral de Seguridad Y Defensa, Documento D.G.P.N. No.07 Mayo. CONFIS.

Muñoz, R., \& Bondia, D. (2009). Victimas Invisibles Conflicto Armado Y Resistencia Civil en Colombia. España: Huygens, ISBN: 978-84-937606-0-1.

Naciones Unidas. (2005). Metodología del marco lógico para la planificación, el seguimiento y la evaluación de proyectos y programas. Santiago de Chile: Naciones Unidas. Acesso em 05 de Junio de 2014, disponível em www.ug.edu.ec: http://www.ug.edu. ec/dipa/senacyt/cepal_manual_marco_logico.pdf 


\section{Referencias}

Naciones Unidas. (2011). Definición del Gasto en Defensa, Seguridad y Justicia. Em Gasto Público en Seguridady Justicia en Centroamérica. Mexico, D.F.: ISSN: 1680-8800.

Otero, A. (2008). Paramilitares La Modernidad que nos Toco. Bogotá D.C., Colombia: Alfonso Otero y Ligia Cortés.

Payá, P. A. (2001). Algunas Reflexiones desde la Sociologia de Max Weber sobre El Control Social y La Disciplina en la Sociedad Actual. Em L. P. Diaz de Leon, La Teoría Sociologica de Max Weber (pp. 85-96). Mexico: UNAM, ISBN 968-369208-07.

Pearce, J. (2005). Policy Failure and Petroleum Depredation: The Economics of Civil war Debate Viewed. Government and Opposition 40(2), 152-80.

Pérez, J., \& Valvuena, G. (2014). La Politica de Seguridad Democratica, 20022006: Efectos Socioeconomicos sobre las areas rurales. Economia Institucional 16(30) pp.241-270.

PNUD. (2011). Colombia Rural Razones para la Esperanza, Informe Nacional de Desarrollo Humano. Bogotá: INDH PNUN, Septiembre: INHD:ISBN: 978-958-8447-63-6.
Romero, M. (2011). La Economia de los Paramilitares. Colombia: Debate.

Semana. (04 de 09 de 2014). A 11 generales los quieren investigar por falsos positivos. Semana. Fonte: http://www.semana. $\mathrm{com} /$ nacion/articulo/por-falsos-positivos-piden-abrir-investigacion-once-generales-del-ejercito/401479-3

Valencia, H. V. (2012). Perspectivas Del Gasto En Seguridad Y Defensa Frente A Cambios En El Conflicto Armado Colombiano (Tesis de Pregrado). Universidad Militar, Bogotá D.C.

Veblen, T. (1965). Teoría de la Empresa de Negocios. EUDEBA.

Weber, M. (1986). El Político y el Cientifico. Madrid: Alianza, Libro de bolsillo num. 71.

Weber, M. (1984). Economia y Sociedad. Mexico: Fondo de CUltura Economica.

Weber, M. (2009 ). La Ética Protestante y el Espíritu del Capitalismo. España: Reus. 